\title{
Immunological correlates of protection from HIV infection and disease
}

\section{Jonathan L Heeney \& Stanley A Plotkin \\ The recent meeting on "Immune Correlates of Protection from HIV Infection and Disease" examined new data from a variety of preclinical and clinical settings. These new insights may facilitate vaccine design and clinical evaluation.}

\begin{abstract}
— or the fifth time in just over 10 years, an F international 'think tank' was held on understanding immune mechanisms that correlate with protection from human immunodeficiency virus 1 (HIV-1) infection and disease ${ }^{1}$. This year's meeting took place immediately before and in conjunction with AIDS Vaccine 2006 in Amsterdam. Many in the HIV vaccine development field have been seeking immune correlates to guide HIV-1 vaccine design and development and to refine clinical trial endpoints. In general terms, there are now three schools of HIV vaccine development. The first, focused on bringing cytotoxic lymphocyte (CTL)-based vaccine candidates to phase III clinical trials, is centered on devel-
\end{abstract} oping T cell-mediated HIV vaccine candidates that will reduce virus load and CD4 T cell loss, prolong survival, and reduce transmission ${ }^{2}$. The challenge of that approach is to generate $\mathrm{CD} 8^{+} \mathrm{T}$ cell responses to conserved HIV-1 epitopes in populations with a diverse HLA background and exposure to a wide variety of HIV-1 genotypes. The second school aims to develop HIV-1 vaccine candidates that will induce broad cross-neutralizing antibodies to conserved B cell epitopes in the HIV-1 envelope $e^{3}$. That has proven extremely difficult, and in the shadow of the failed phase III Vaxgen trials of viral envelope (Env), there are relatively

Jonathan L. Heeney is in the Department of Virology, Biomedical Primate Research Centre, 2288 GJ Rijswijk, and the University of Leiden, The Netherlands. Stanley A. Plotkin is with Sanofi Pasteur, 69367 Lyon, France, and the University of Pennsylvania, Philadelphia, Pennsylvania 19104, USA.

e-mail: heeney@bprc.nl few vaccine candidates of that type in clinical trial. The third school believes a combined approach will be necessary, in which broad Thelper responses should be induced to drive $B$ cell responses to conserved neutralizing epitopes and CTL targets of HIV ${ }^{4}$.

Insights into cell-mediated control of HIV The outcome of HIV-1 infection in human populations is variable and greatly influenced by HLA diversity. For example, a relatively small number of people infected with HIV-1 for 10 years or more effectively control infection. In this population of 'natural human controllers', people with HLA-B57 and HLA-B27 are overrepresented. CTL epitopes restricted by those alleles are in highly conserved regions of the HIV-1 group-associated antigen (Gag) protein. Mutations in the genes encoding those epitopes often extract a 'fitness cost' from the virus and therefore produce a lower viral load in the patient ${ }^{5,6}$. It is becoming apparent that the HIV-1 CD8 ${ }^{+} \mathrm{T}$ cell response is complex and is under-represented when interferon- $\gamma($ IFN- $\gamma$ ) alone is measured and that the quality of the response is more important than its magnitude (Fig. 1a). Polyfunctional CD8 ${ }^{+} \mathrm{T}$ cell responses defined by expression of combinations of four or five cytokines, chemokines and functional markers (such as IFN- $\gamma$, interleukin 2 (IL-2), CD107, macrophage inflammatory protein $1 \beta$ (MIP-1 $\beta$ ) and tumor necrosis factor) correlate with improved control of viral load (Richard Koup, Bethesda, Maryland, USA) ${ }^{7}$.

Such responses seem to depend on competent IL-2-producing $\mathrm{CD} 4^{+} \mathrm{T}$ cells, which correlates with the maintenance of $\mathrm{T}$ cell proliferative responses ${ }^{8,9}$ (Mark Connors, Bethesda, Maryland, USA; Fig. 1b). In HIV-2, as in HIV-1, infection nonprogression or slow progression is correlated with qualitative $\mathrm{CD} 4^{+}$ Thelper responses, presumably also important for maintaining effector $\mathrm{CD} 8^{+} \mathrm{T}$ cell responses and control of virus load (Sarah RowlandJones, Fajara, Gambia) ${ }^{10}$. However, cause and effect must be differentiated, as maintenance of effector $\mathrm{CD}^{+} \mathrm{T}$ cell responses may be due to a less pathogenic HIV-2 infection. There is general consensus that the impairment of $\mathrm{CD}^{+}$ CTLs in progressive HIV disease is due mainly to loss of $\mathrm{CD}^{+} \mathrm{T}$ cell help ${ }^{4,11}$; that has been correlated with loss of HIV-1-specific IL-2 ${ }^{+}$ and IFN- $\gamma^{+} \mathrm{IL}-2^{+} \mathrm{CD} 4^{+} \mathrm{T}$ cells of the central memory phenotype ${ }^{12,13}$.

Consistent with earlier reports of increased anergy and apoptosis, the cell surface receptor $\mathrm{PD}-1$ is expressed on $\mathrm{HIV}-1$-specific $\mathrm{CD}^{+}$and $\mathrm{CD} 4^{+} \mathrm{T}$ cells in HIV-infected people who progress to AIDS, and this correlates with plasma virus loads ${ }^{14-16}$. Giuseppe Pantaleo (Lausanne, Switzerland) has found that in people who control virus load, polyfunctional $\mathrm{CD}^{+} \mathrm{T}$ cells are driven mainly by virus epitopes restricted by specific HLA-B alleles (such as HLA-B ${ }^{\star} 57$, HLA $-B^{\star} 27$ and HLA-B $\left.{ }^{\star} 5701\right)^{17}$. Those findings emphasize the effect that HLA diversity has in HIV-infected populations.

Studies by Mary Carrington (Frederick, Maryland, USA) of large genetic cohorts of HIV-1-infected people have demonstrated a survival benefit of certain HLA-B alleles ${ }^{18}$. HLA-B proteins can be separated into two large groups: BW6 (representing about 60\%), which are not ligands for killer-inhibitory receptors (KIRs), and BW4 (40\%), of which -80I and $-80 \mathrm{~T}\left(\mathrm{HLA}^{\star}{ }^{\star} 27\right)$ are alleles, are KIR3DL1 ligands. HLA-BW4 interacts with KIR3DL1 allotypes and is associated with more protection against $\mathrm{CD} 4^{+} \mathrm{T}$ cell loss and lower virus load than is HLA-BW6 (ref. 18). However, the 
a Monospecific IFN- $\gamma \mathrm{CD} 8^{+} \mathrm{CTL}$

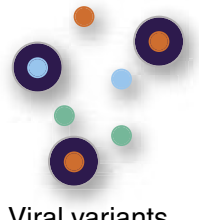

Viral variants
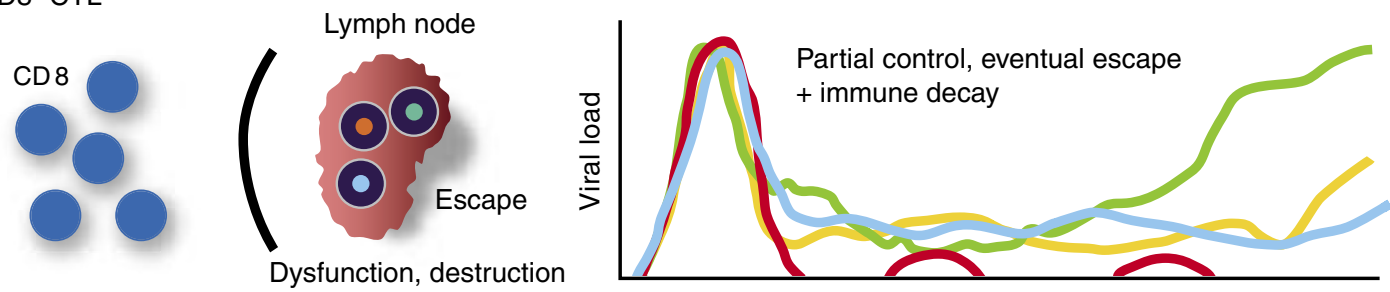

b Polyfunctional and antigenically broad CTLs \& T helper cells
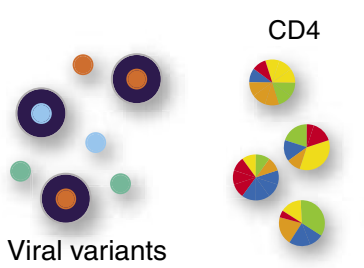
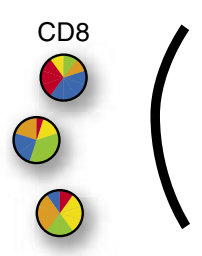

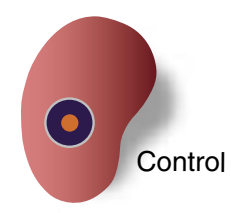

Adaptive response

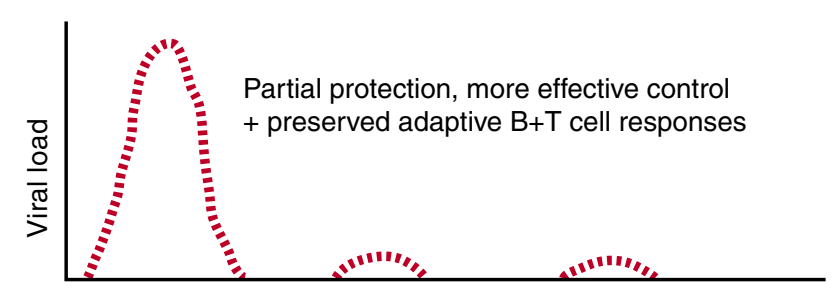

Figure 1 The effect of quantitative versus qualitative T cell responses and the control of HIV infection. (a) Monospecific IFN- $\gamma$ CD8 ${ }^{+} \mathrm{T}$ cell responses (blue cells) are associated with less effective control of viral replication and, ultimately, viral escape (virions are small dots of different colors corresponding to different lines of viremia; right), followed by immune decay (lymph nodes) and suppression. (b) Polyfunctional and antigenically broad HIV-1-specific CD8 ${ }^{+}$ CTL and IL-2-rich CD4+ $\mathrm{T}$ helper cell responses. Evidence suggests that HIV-specific $\mathrm{CD}^{+}$and $\mathrm{CD} 4^{+} \mathrm{T}$ cell responses, polyfunctional in terms of cytokines, chemokines and cytolytic markers (multicolored circles), are associated with better control of HIV viral loads. Control of HIV viral loads is also associated with T cell proliferation and rigorous $\mathrm{CD} 4^{+} \mathrm{T}$ cell responses characterized by IL-2 as well as IFN- $\gamma$ production. B+T cell, B cell plus $\mathrm{T}$ cell.

specific mechanism of that enhanced natural killer (NK) cell activity is not yet understood. Obviously, most $\mathrm{CD}^{+} \mathrm{T}$ cell responses in chronic HIV infection are not associated with effective control of viremia ${ }^{19}$. Instead, protection may be correlated with responses targeted to the HIV protein Gag (Philip Goulder, Oxford). Data from a large cohort in Durban, South Africa, suggest that anti-Gag $\mathrm{CD} 8^{+} \mathrm{T}$ cell responses may be better correlated with control of virus load than with individual HLA associations.

\section{AIDS-resistant nonhuman primates}

More than 30 identified primate species that live on the African continent are infected with 'simian immunodeficiency viruses' (SIVs) ${ }^{20}$. In their natural hosts, SIVs do not usually cause disease. However, when SIVsm (a strain from sooty mangabeys) infects Asian rhesus monkeys (Macaca species), it causes an AIDS-like disease similar to AIDS caused by HIV-1 in humans ${ }^{21}$. The HIV-1 $\mathrm{M}$ and $\mathrm{N}$ groups originated from the chimpanzee SIV strain SIVcpz, whereas HIV-2 originated from SIV $\mathrm{sm}^{21}$. The mechanisms of natural resistance to AIDS have been studied in three species, chimpanzees (Pan troglodytes), sooty mangabeys (Cercocebus atys) and African green monkeys (Cercopithecus aethiops sabaeus). As reviewed by Erik Rutjens (Rijswijk, The Netherlands) chimpanzees infected with HIV-1 or SIVcpz are relatively more resistant to AIDS than are humans, not because they control virus load better but because of reduced immunopathology $y^{21,22}$. NK cells, one of the chief effectors of innate-like immunity, are found in much greater numbers in the circulation in chimpanzees than in humans. Chimpanzees are able to maintain intact NK cell cytolytic and regulatory cytokine functions in the face of chronic viremia, and in contrast to humans, they are also able to differentially regulate NK cell receptors that have important dendritic cell editing capabilities. Mark Feinberg (Atlanta, USA) demonstrated that plasmacytoid dendritic cells from sooty mangabeys have a lower response to type 1 interferons than do AIDSsusceptible rhesus monkeys and humans ${ }^{23}$. Studying infection with the virus SIVagm, Michaela Muller-Trutwin (Paris) similarly compared the responses of AIDS-susceptible rhesus macaques to those of infected asymptomatic African green monkeys. Although T cell activation markers and certain 'proinflammatory' cytokines (IFN- $\alpha$, IFN- $\gamma$ and transforming growth factor- $\beta$ ) were present in the acute phase in both species, African green monkeys did not have measurable tumor necrosis factor and had only a weak IFN- $\gamma$ response. Notably, expression of IL-10, transcription factor Foxp 3 and transforming growth factor- $\beta_{1}$ was detected earlier in acute infection, suggestive of a function for regulatory $\mathrm{T}$ cells in controlling chronic immune activation in African green monkeys ${ }^{24,25}$.

As an alternative hypothesis, natural resistance to AIDS may simply be related to tar- get cell availability. Ron Veazey (Covington, Louisiana, USA) presented data showing that naturally resistant SIV-infected monkeys have fewer $\mathrm{CD} 4^{+} \mathrm{T}$ cells or lower CCR5 expression on $\mathrm{CD}^{+} \mathrm{T}$ cells $\mathrm{s}^{26,27}$. Notably, whereas both AIDS-susceptible and AIDS-resistant hosts developed an acute loss of $\mathrm{CD} 4^{+} \mathrm{CCR} 5^{+}$ memory $\mathrm{T}$ cells in the intestinal mucosa, those were (in part) mostly restored in disease-resistant monkeys and as noted peripherally in chimpanzees $^{28}$.

\section{Protection from lentivirus infection?}

Passive transfer of monoclonal antibodies and immune sera in rhesus monkeys and similar earlier studies in chimpanzees has provided proof that protection from infection can be mediated by antibodies ${ }^{29}$. However, the issue remains of whether antibody-based vaccine protection can be achieved at urogenital mucosal surfaces. Data from highly exposed commercial sex workers in Kenya and elsewhere suggest that several mechanisms (such as antibodies, $\mathrm{CD}^{+} \mathrm{T}$ cells, CTLs and so on) might be important in protection from heterosexual transmission ${ }^{30}$. In a prospectively studied cohort of HIV-1-serodiscordant heterosexual couples established in Uganda, F. Gotch (London) found that CD91 (heatshock protein receptor) expression on $\mathrm{CD} 14^{+}$ monocytes was much higher in 'exposed seronegative' people than in their seropositive partners ${ }^{31}$, suggesting an abortive or limited infection. 
Chris J. Miller (Davis, California, USA) described a series of studies in macaques in which repeated vaginal exposure with relatively low doses of SIVmac251, high doses of SIVmac239 or attenuated derivates were administered to determine in which circumstances protection was obtained. Repeated low-dose vaginal exposure did not lead to a protective state but instead eventually led to systemic infection ${ }^{32,33}$.

글 none of those settings was there evidence of adaptive cellular or humoral immune responses, but there was increased expression of innate antiviral immune responses and proinflammatory markers (IL-10, transforming growth factor- $\beta$ and Foxp 3 in vagina and cervix; IFN- $\alpha$ in draining lymph nodes) in exposed, apparently uninfected macaques. Disappointingly, efforts to induce innate responses by Toll-like receptor ligands failed to induce protection from vaginal exposure in the macaque mode ${ }^{34}$. In a prophylactic vaccine efficacy studies using DNA priming-modified vaccinia virus Ankara boosting, all naive macaques became infected after a maximum of eight exposures to low doses of virus (one per week), whereas $70 \%$ of vaccinees were infected, but only after as many as eighteen doses of virus ${ }^{35}$. Sal Butera (Atlanta, USA) reviewed how the repeated low-dose exposure model has been used to evaluate susceptibility to infection and vaccine candidates. Although the low-dose exposure model may better model human exposure, protection induced by mucosal (rather than from parenteral) vaccination has not been readily achieved.

\section{Preclinical vaccine efficacy studies}

The SIV and SHIV (HIV-SIV chimeras) models of AIDS offer the potential for unraveling the immune correlates of vaccine protection when careful immune monitoring is followed by vaccine challenge in well controlled settings. Gerrit Koopman (Rijswijk, The Netherlands) reviewed many DNA prime-boost HIV-1 vaccine studies in monkeys. In the few studies in which protection from infection has been noted, neutralizing antibodies to Env have occasionally been found. In settings evaluating $\mathrm{T}$ cell-based vaccine strategies for control of virus load, the most rigorous vaccine responses were directed against the Gag antigen, which induced substantial proliferative as well as IFN- $\gamma$ and IL-2 responses, as assessed by enzyme-linked immunospot assay (ELISpot), in contrast to Env, which induced somewhat weaker IFN- $\gamma$ and IL-2 responses but a substantial IL-4 response, as assessed by ELISpot assay. Protection from CD4 ${ }^{+} \mathrm{T}$ cell loss was often seen when the $\mathrm{T}$ cell response to Gag was dominant. However in the absence of Gag, improved DNA plasmids encoding Env, Tat and negative factor (Nef), when delivered by gene

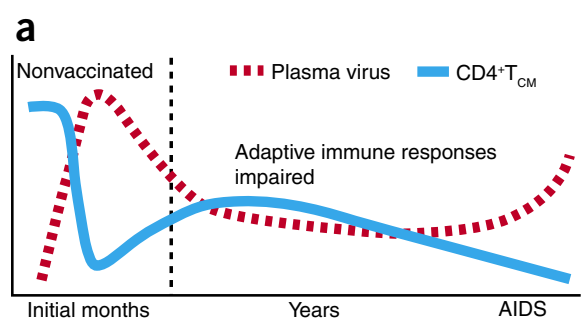

b
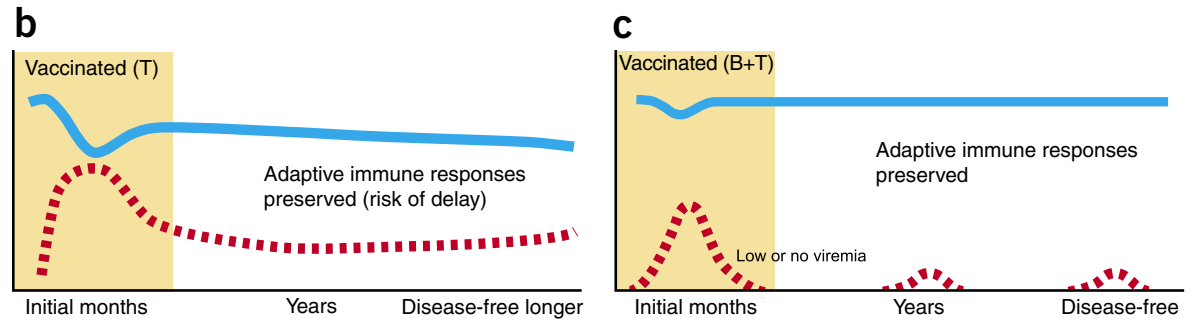

Figure 2 Outcome of HIV infection in the absence or presence of vaccination. (a) Substantial loss of central memory T cells $\left(T_{c m}\right)$ early in HIV infection in people who lack any pre-existing immunity. (b) Potential to bridge the acute $\mathrm{CD}^{+}{ }^{+}$central memory $\mathrm{T}$ cell loss using $\mathrm{T}$ cell-based ( $\mathrm{T}$ helper cell and CTL) vaccine candidates (such as Ad5 or DNA-NYVAC), thus providing an intact dendritic cell-T helper cell environment for continued adaptive B cell and T cell responses. T, T cell. (c) Vaccine-induced HIVneutralizing antibody responses (optimally, soluble immunoglobulin $A$ ) at mucosal surfaces. B+T, B cell and $\mathrm{T}$ cell.

gun, generated 'preferential' IFN- $\gamma$ and IL-2 responses to the proteins encoded, whereas when primed or boosted with protein, IL-4 $\mathrm{CD}^{+} \mathrm{T}$ cell responses became predominant. Retrospective comparison of 12 different strategies showed that IL-2 $\mathrm{CD} 4^{+}$responses were best preserved after pathogenic SHIV challenge when Gag was included in the vaccine candidates and that this correlated with the best long-term protection from $\mathrm{CD} 4^{+} \mathrm{T}$ cell loss. That conclusion was supported by results presented by Marie-Claire Gauduin (San Antonio, Texas, USA). Live attenuated SIV strains were found to induce a high frequency of broadly directed SIV-specific $\mathrm{CD} 4^{+} \mathrm{T}$ cells. $T$ cell vaccine strategies without Env have also been evaluated. David Watkins and colleagues (Madison, Wisconsin, USA) studied $\mathrm{CD}^{+} \mathrm{T}$ cell responses induced by a DNA-adenovirus (Ad5) vaccine candidate encoding Gag, Tat, Rev and Nef proteins. Partial control of SIV $_{\text {MAC239 }}$ for up to a year after challenge was attributed to strong $\mathrm{CD}^{+} \mathrm{T}$ cell responses to Gag, Nef, Tat and Rev.

In a larger comprehensive DNA-Ad5 vaccine study designed to evaluate the efficacy of Gag-Pol (polymerase), Env alone, Gag-Pol plus Env or Ad5 alone, Gary Nabel (Bethesda, Maryland, USA) and colleagues found that SIV RNA plasma virus loads were controlled better in vaccinees than in controls. The most important correlate of survival seemed to be not the vaccine antigens but instead the persistence of $\mathrm{CD}^{+}{ }^{+}$central memory $\mathrm{T}$ cell counts ${ }^{36}$ and $\mathrm{CD} 8^{+} \mathrm{T}$ cell responses (that is, Gag-specific
$\mathrm{CD}^{+}$central memory $\mathrm{T}$ cells but not $\mathrm{CD} 8^{+}$ effector memory $\mathrm{T}$ cells). Mario Roederer (Bethesda, Maryland, USA) of the same institute drove home the point that measurement of total CD4 T cells underestimates viral effects. It is the central memory $\mathrm{T}$ cell phenotype that is substantially and consistently affected in acute infection, particularly in the lamina propria of the intestine. Estimates show that as many as $60 \%$ of all CD 4 memory $T$ cells may be infected at a single time point, and by day 14 after infection, $80 \%$ of all infected cells are lost (approximately $50 \%$ of all memory CD4 ${ }^{+} \mathrm{T}$ cells in 4 days; Fig. 2a). Notably, data indicate that systemic vaccination can (in part) protect from that considerable loss of $\mathrm{CD} 4^{+}$memory $\mathrm{T}$ cells in the acute period in mucosal as well as other compartments $^{37}$ (Fig. 2b). Thus, certain candidate vaccines afford vaccinated macaques a degree of protection from CD4 T cell loss, and that correlates with a robust multifunctional $\mathrm{T}$ cell response. The remaining issues include whether these same vaccine candidates will induce similar types of responses and whether they will be predictive of vaccine efficacy in humans.

\section{T cell responses in immunized volunteers}

So far, more than 4,000 human volunteers have been enrolled by the Human Vaccine Trials Network alone. An overview presented by Julie McElrath (Seattle, USA) ranked vaccine candidates according to the percentage of responders they elicited. Of the candidates they studied, adenovirus-based vectors with 
or without DNA priming gave the highest percentage of responders. Richard Koup presented data on the 'DNA-rAd5' vaccine candidate of the Vaccine Research Center of the National Institutes of Health, assessed by 12-color flow cytometry. Like the four or five polyfunctional responses noted in human long-term nonprogressors, volunteers immunized with DNA-Ad5 had strong multifunctional $\mathrm{CD} 4^{+}$ and $\mathrm{CD} 8^{+} \mathrm{T}$ cell responses that were broadly responsive to many vaccine-encoded antigens. The 'DNA-NYVAC' candidate of the European EuroVacc Foundation also induced a high degree of $\mathrm{CD}^{+} \mathrm{T}$ cell responses that were polyfunctional and were associated with much higher IFN- $\gamma$ production per cell (Richard Koup).

Characteristics of the immune responses elicited by the Merck Ad5 HIV (Gag, Pol, Nef) vaccine candidate were presented by Danilo Casimiro (West Point, Pennsylvania USA). Most subjects had $\mathrm{T}$ cell responses to the clade B vaccine candidate $(>70 \%$ at a viral particle dose of $\left.3 \times 10^{10}\right)$. The responses were robust and cross-reactive with near-consensus protein sequences from other clades $(\mathrm{Pol}>\mathrm{Gag}>$ $\mathrm{Nef})$. The $\mathrm{CD} 8^{+} \mathrm{T}$ cell responses in the vaccine recipients were of reasonable breadth in terms of responses to all three antigens.

\section{Vaccine-elicited effector antibodies}

The importance of antibody Fc $\gamma$ receptor interactions on monocytes-macrophages and NK cells $\left(\mathrm{CD} 16^{+}\right)$for eliciting functionaleffector responses was demonstrated by Don Forthal (Irvine, California, USA). Antibodydependent cell-mediated virus inhibition correlated inversely with viral load during acute HIV infection in humans ${ }^{38}$ and prevented neonatal SIV infection, but did not correlate with virus neutralization. In the context of replication-competent adenovirus vector immunization, passively transferred immunoglobulin $\mathrm{G}$ with antibody-dependent cellular cytotoxicity activity did not protect neonatal macaques (Marjorie Robert-Guroff, Bethesda, Maryland, USA). Susan Barnett (Emeryville, California, USA) reported studies in which Env antigen was delivered by the alphavirus chimera VEE-SIN or as recombinant Env protein with adjuvant, presented in various systemic and/or mucosal prime-boost vaccine combinations followed by mucosal challenge of macaques with the chimeric virus SHIVsf162. Unexpectedly, the highest neutralizing antibody titers before challenge and lowest viral loads after rectal challenge were not obtained when the mucosal site was primed (intranasally or intrarectally) by VEE-SIN Env and boosted intramuscularly, but instead when both doses were given intramuscularly. Moreover, when 'adjuvanted' Env protein vaccine was administered by either the intranasal or intramuscular route or a combination of those routes, Env-specific immunoglobulin A in vaginal washes was found more often in monkeys protected from vaginal challenge. The most optimal mucosal immunogen combinations for inducing broad neutralizing antibodies have not yet been identified, but studies such as these are providing greater insight into how to induce better mucosal lentiviral immunity.

\section{Conclusions}

At this meeting there was a palpable sense that the definition of immune correlates of protection has moved forward. The destruction of central memory T cells, observed most notably in the intestinal mucosa, seems to be the main prognostic factor for progression of disease, and therefore its prevention now represents a goal of vaccination. Eliciting neutralizing antibodies remains an important but difficult objective. However, non-neutralizing antibodies may provide some protection against infection in certain cases. In humans and AIDS-resistant monkeys, absence of immune activation is a protective correlate. Data now indicate that a successful $\mathrm{T}$ cell vaccine must stimulate polyfunctional $\mathrm{CD}^{+}$as well as $\mathrm{CD}^{+} \mathrm{T}$ cells capable of secreting IL- 2 and other cytokines. Some vaccine candidates now in trial may be capable of achieving that goal. However, the importance of protection at the mucosa is apparent (Fig. 2c), and much needs to be learned about immune responses acting at the most common sites of infection.

\section{ACKNOWLEDGMENTS}

We thank T. de Koning, H. van Westbroek,

N. Beenhaaker and P. Mooij for assistance; B. Haynes, R. Weiss, L. Corey for chairing discussions; and all of the participants for the high quality of cutting-edge science. We regret omission of persons and data owing to space limitations.

1. Heeney, J.L. et al. Immunol. Today 18, 4-8 (1997).

2. McMichael, A.J. Annu. Rev. Immunol. 24, 227-255 (2006).

3. Burton, D.R. et al. Nat. Immunol. 5, 233-236 (2004)

4. Heeney, J.L. Expert Rev. Vaccines 3, S53-S64 (2004).

5. Gao, X. et al. Nat. Med. 11, 1290-1292 (2005).

6. Goulder, P.J. \& Watkins, D.I. Nat. Rev. Immunol. 4 630-640 (2004).

7. Betts, M.R. et al. Blood 107, 4781-4789 (2006).

8. Iyasere, C. et al. J. Virol. 77, 10900-10909 (2003).

9. Lichterfeld, M. et al. J. Exp. Med. 200, 701-712 (2004).

10. Duvall, M.G. et al. J. Immunol. 176, 6973-6981 (2006).

11. Gougeon, M.L. et al. J. Immunol. 158, 2964-2976 (1997).

12. Boaz, M.J., Waters, A., Murad, S., Easterbrook, P.J. \& Vyakarnam, A. J. Immunol. 169, 6376-6385 (2002).

13. Harari, A., Vallelian, F., Meylan, P.R. \& Pantaleo, G. J. Immunol. 174, 1037-1045 (2005).

14. Day, C.L. et al. Nature 443, 350-354 (2006).

15. Petrovas, C. et al. J. Exp. Med. 203, 2281-2291 (2006).

16. Trautmann, L. et al. Nat. Med. 12, 1198-1202 (2006).

17. Kiepiela, P. et al. Nature 432, 769-775 (2004).

18. Qi, Y. et al. PLoS Pathog. 2, e79 (2006).

19. Lichterfeld, M., Yu, X.G., Le Gall, S. \& Altfeld, M. Trends Immunol. 26, 166-171 (2005).

20. Aghokeng, A.F. \& Peeters, M. J. Neurovirol. 11, 27-32 (2005).

21. Heeney, J.L., Dalgleish, A.G. \& Weiss, R.A. Science 313 462-466 (2006).

22. Rutjens, E. et al. Front. Biosci. 8, d1134-d1145 (2003).

23. Dunham, R. et al. Blood 108, 209-217 (2006).

24. Kornfeld, C. et al. J. Clin. Invest. 115, 1082-1091 (2005).

25. Ploquin, M.J. et al. Retrovirology 3, 37-42 (2006).

26. Pandrea, I. et al. Blood published online 26 September 2006 (doi:10.1182/blood-2006-05-02364).

27. Veazey, R.S. et al. Science 280, 427-431 (1998).

28. Heeney, J.L. Immunol. Today 16, 515-520 (1995).

29. Mascola, J.R. Curr. Mol. Med. 3, 209-216 (2003).

30. Shacklett, B.L. Curr. HIV/AIDS Rep. 3, 26-31 (2006).

31. Kebba, A. et al. J. Leukoc. Biol. 78, 37-42 (2005)

32. Ma, Z.M., Abel, K., Rourke, T., Wang, Y. \& Miller, C.J. J. Virol. 78, 14048-14052 (2004).

33. McDermott, A.B. et al. RJ. Virol. 78, 3140-3144 (2004).

34. Wang, Y. et al. J. Virol. 79, 14355-14370 (2005).

35. Ellenberger, D. et al. Virology 352, 216-225 (2006).

36. Letvin, N.L. et al. Science 312, 1530-1533 (2006).

37. Mattapallil, J.J. et al. J. Exp. Med. 203, 1533-1541 (2006).

38. Forthal, D.N., Landucci, G., Phan, T.B. \& Becerra, J. J. Virol. 79, 2042-2049 (2005) 